\title{
Heterotic Models of Aggregate Demand
}

\author{
By Gael Giraud and John K.-H. Quah
}

ABSTRACT: A common theme in the theory of demand aggregation is that market demand can acquire properties which are not always individually present among the agents who make up that market, a phenomenon we call heterosis in this paper. This paper focusses on the well known result that with a suitable distribution of demand behavior (arising perhaps from the underlying distribution of preferences), market demand can become approximately a linear function of income or even take on approximate Cobb-Douglas properties. We highlight the mathematical arguments underpinning these models and show that in the right context, it is possible to carry the arguments further and achieve exact rather than just approximate results: exact Cobb-Douglas market demand or exact linearity of market demand with respect to income.

KEYwORDS: heterosis, heterogeneity, Cobb-Douglas, homotheticity, law of demand, aggregation

JEL Classification Nos: D11, D50.

\section{AdDREsSES:}

Gael Giraud, CNRS UMR 7522, Bureau d'Economie Theorique et Appliquee, 61, avenue de la Foret Noire, 67000, Strasbourg, France; ggiraud@cournot.u-strasbg.fr John Quah, St Hugh's College, Oxford, OX2 6LE, UK; john.quah@economics.ox.ac.uk 


\section{INTRODUCTION}

CONSIDER A MARKET consisting of agents with different demand functions or correspondences and different incomes. The typical question in demand aggregation is to ask for the conditions which will guarantee that aggregate demand takes on a particular property.

There are two mutually non-exclusive approaches to problems of this sort. The more obvious way is simply to ask for the conditions on individual demand which will guarantee that the aggregate property holds. This approach is most useful when the property required is straightforwardly aggregable. So one way of ensuring that aggregate demand is a continuous function of price is to assume that individual demand is a continuous function of price, which in turn follows from preferences which are continuous and strictly convex. Sometimes the solution is less straightforward. For example, it is well known that aggregate demand need not satisfy the strong or weak axioms, even when the agents who make up that market satisfy those properties. In these instances, stronger properties have to be imposed at the individual level to obtain the desired aggregate properties. For example, the strong axiom (essentially the existence of a representative consumer) is guaranteed if all agents have preferences representable by the Gorman polar form; the weak axiom in the aggregate holds provided agents individually obey the law of demand.

This approach to aggregation problems is distinguished by two features: the property required at the individual level are at least as strong as the aggregate property required and once it is satisfied, the precise distribution of demand and income characteristics does not matter. The focus of this paper is on another approach in which these features are reversed. In this second approach, the assumptions made at the individual level are weaker than, or 
at least different from, the aggregate property required, and the aggregate property arises because of the distributional assumptions imposed. This approach is sufficiently common that it is worth giving it a name. We propose that it be called heterosis. The suffix '-osis', following the Greek, is used in English to denote a process or condition: heterosis refers to the phenomenon in which the distributional characteristics, i.e., heterogeneity, of a market, imbues market demand with properties which are not always present at the individual level. ${ }^{1}$

Examples of heterosis abound. An application of Lyapunov's theorem guarantees that when the measure space of agents is atomless, the aggregate demand correspondence is convex valued, even when each type of agent may not have a convex valued demand correspondence (see Hildenbrand (1974)). Beyond this, there is a substantial literature on when a market will have a continuous or smooth demand function, even when non-convexities are present at the individual level (see, for example, Dierker et al (1980)). Another well known example of heterosis is the result due to Hildenbrand (1983). It says that a market in which all agents have the same demand function which obeys the weak axiom will satisfy a stronger property - the law of demand - provided the income distribution has a downward sloping density function.

The focus of this paper is the family of heterotic models first studied by Grandmont (1992). Grandmont considers a market consisting of agents with the same income and different demand functions belonging to the same affine equivalence class. Affine transformations and equivalence classes are defined in Section 3; what should be noted here is that affine transformations preserve rationality properties like the weak and strong axioms, so that Grandmont's model has the important feature that all agents in the market can satisfy 
the classical rationality restrictions, even though these restrictions are not required for his aggregation result. Assuming that there are $l$ goods in this market, demand functions can then be parametrized by elements in $R^{l}$. When the density function on the parameter is sufficiently flat in some precise sense, market demand becomes increasingly Cobb-Douglas like, i.e., the proportion of market expenditure devoted each good is approximately constant.

A model closely related to Grandmont's is the heterotic model of Quah (1997). By modifying the parametric assumptions in Grandmont (1997), Quah identifies the conditions under which a market will take on homothetic-like (but not necessarily Cobb-Douglaslike) properties; in particular, aggregate demand is approximately linear in income and the aggregate of income effects is approximately positive semi-definite.

Section 2 of this paper is a careful re-examination of the mathematical features common to these models. We show that what underlies these results is really a theorem about translations of a function, which can be stated in the following way: a translation of the function $f: R^{l} \rightarrow R^{m}$ is another function $f_{t}$, where $t$ is in $R^{l}$ and $f_{t}(x)=f(x+t)$; under certain assumptions, on any bounded subset $X$ of $R^{l}$, there exists density functions $h$ such that $F(x)=\int_{R^{l}} f_{t}(x) h(t) d t$ becomes approximately independent of $x$ in $X$. The main mathematical result of this paper is that by endowing the set of functions with a suitable topology and with certain additional assumptions, the closure of the set of translations of $f$ becomes a compact set. On this compact set of functions, there exists a distribution which guarantees that the average function is a constant, i.e., exactly independent of $x$.

After establishing this mathematical result in Section 2, we go on to apply it in Section 3. We identify the assumptions which guarantee that the distribution of demand is such 
that the market's average expenditure share on each good is independent of prices, i.e., the market demand function behaves as though it is generated by a Cobb-Douglas preference. This result is therefore an exact version of Grandmont's theorem. We then apply the result to Quah's model, where we identify distributional conditions under which market demand becomes exactly linear in income and the aggregate of income effects is exactly positive semi-definite. Section 3 also includes a brief discussion of the relationship between this paper and the exact aggregation results in Giraud and Maret (2001).

An issue which has attracted some debate recently is the precise nature of the heterosis at work in Grandmont's model. Hildenbrand and Kneip (1999) have a model in which agents' individual behavior are allowed to depart from the Cobb-Douglas form, but large departures occur for different agents at different parts of the price space (hence the "heterogeneity" in the title of their paper). This has the effect that at any single price vector, only a small fraction of agents actually have large deviations from Cobb-Douglas behavior and so CobbDouglas behavior holds approximately in aggregate. They show that Grandmont's model, with some additional assumptions, can be understood as an example of heterosis in this sense. ${ }^{2}$ This is a legitimate view of the mechanism at work behind Grandmont's theorem, but it is not the only possible view. In particular we show in Section 4 that the aggregate Cobb-Douglas behavior guaranteed by the theorem could arise from complementary or signbalancing heterogeneity. (The terms are loosely borrowed from B. de Villemeur (2001) and Hildebrand and Kneip (1999) respectively.) In other words, the average expenditure share on each good is approximately constant because, while some agents may increase their expenditure share on a good as prices change, this is perfectly balanced by other agents 
who choose to reduce their expenditure share on the same good. This is unlike the situation depicted in Hildenbrand and Kneip (1999) because the set of agents who deviate significantly from Cobb-Douglas behavior at each price need not be small.

\section{The Main Results}

Let $P$ be a set and consider a function $\bar{s}: P \times R^{l} \rightarrow R^{m}$. With any $t$ in $R^{l}$, we can define $s_{t}$, a function from $P \times R^{l}$ to $R^{m}$ by $s_{t}(p, x)=\bar{s}(p, x+t)$. So $s_{t}$ is just a translation of $\bar{s}$. We denote by $\mathcal{S}$ the set $\left\{s_{t}: t \in R^{l}\right\}$. If $\bar{s}(p, \cdot)$ is measurable and bounded, then for any density function $h: R^{l} \rightarrow R_{+}$, the integral $\int s_{t}(p, x) h(t) d t$ exists; abusing notation somewhat, we will denote it by $s_{h}(p, x)$. So $s_{h}(p, x)$ is the average value of $s_{t}(p, x)$ when $t$ is distributed according to $h$. The next result is essentially due to Grandmont (1992) and is at the heart of his aggregation results.

Proposition 2.1. Suppose $\bar{s}(p, \cdot)$ is measurable and $\left|\bar{s}^{j}(p, \cdot)\right|$ is bounded by $M$. If the density function $h$ is $C^{1}$, then

$$
\left|s_{h}^{j}(p, x)-s_{h}^{j}(p, \tilde{x})\right| \leq M B(h) \max _{1 \leq i \leq l}\left\{\left|x^{i}-\tilde{x}^{i}\right|\right\}
$$

where $B(h)=\sum_{i=1}^{l} \int_{R^{l}}\left|\partial h / \partial t^{i}\right| d t$.

Proof: Note that $s_{h}(p, \tilde{x})=\int_{R^{l}} \bar{s}(p, \tilde{x}+t) h(t) d t=\int_{R^{l}} \bar{s}(p, x+t) h(t+x-\tilde{x}) d t$. Therefore,

$$
\begin{aligned}
\left|s_{h}^{j}(p, x)-s_{h}^{j}(p, \tilde{x})\right| & \leq\left|\int_{R^{l}} \bar{s}^{j}(p, t+x)(h(t)-h(t+x-\tilde{x})) d t\right| \\
& \leq M \int_{R^{l}}|h(t)-h(t+x-\tilde{x})| d t .
\end{aligned}
$$

Writing $x-\tilde{x}$ as $\alpha$, we see that $\int_{R^{l}}|h(t)-h(t+\alpha)| d t$ is bounded by

$$
\sum_{i=1}^{l} \int_{R^{l}}\left|h\left(t^{1}+\alpha^{1}, t^{2}+\alpha^{2}, \ldots, t^{i-1}+\alpha^{i-1}, t^{i}, \ldots, t^{l}\right)-h\left(t^{1}+\alpha^{1}, t^{2}+\alpha^{2}, \ldots, t^{i}+\alpha^{i}, t^{i+1}, \ldots, t^{l}\right)\right| d t
$$




$$
\begin{aligned}
& =\sum_{i=1}^{l} \int_{R^{l}}\left|\int_{0}^{\left|\alpha^{i}\right|} \frac{\partial h}{\partial t^{i}}\left(t^{1}+\alpha^{1}, t^{2}+\alpha^{2}, \ldots, t^{i}+y^{i}, t^{i+1}, \ldots, t^{l}\right) d y^{i}\right| d t \\
& \leq \sum_{i=1}^{l} \int_{0}^{\left|\alpha^{i}\right|} \int_{R^{l}}\left|\frac{\partial h}{\partial t^{i}}\left(t^{1}+\alpha^{1}, t^{2}+\alpha^{2}, \ldots, t^{i}+y^{i}, t^{i+1}, \ldots, t^{l}\right)\right| d t d y^{i} \\
& =\sum_{i=1}^{l}\left|\alpha^{i}\right| \int_{R^{l}}\left|\frac{\partial h}{\partial t^{i}}(t)\right| d t .
\end{aligned}
$$

This last term is in turn bounded by $B(h) \max _{1 \leq i \leq l}\left\{\left|x^{i}-\tilde{x}^{i}\right|\right\}$.

The interesting thing to note in Proposition 2.1 is that when $B(h)$ is small, $\mid s_{h}^{j}(p, x)-$ $s_{h}^{j}(p, \tilde{x}) \mid$ becomes small as well; more precisely, on any bounded subset of $R^{l}, s_{h}^{j}(p, \cdot)$ will become increasingly independent of $x$ if $h$ can be found such that $B(h)$ is arbitrarily small. It is not hard to see that such density functions exist. First we choose any density function $h$ such that $B(h)$ is finite. Then one can easily verify that for the sequence of density functions $\left\{h_{n}\right\}_{n \geq 1}$ where $h_{n}(t)=n^{-1} h\left(n^{-1} t\right), B\left(h_{n}\right)$ tends to zero as $n$ tends to infinity.

What we aim to do is to establish the existence of a distribution on $\mathcal{S}$ (or, as it turns out, on a slightly larger set) such that its average value becomes exactly, rather than just approximately, independent of $x$. To do this, we need to impose more structure on the problem.

We assume that $P$ is a metric space; we also assume that it is $\sigma$-compact, by which we mean that there is a sequence of compact sets $\left\{P_{n}\right\}_{n \geq 1}$ such that $\cup_{n=1}^{\infty} P_{n}=P$ and $P_{n} \subset P_{n+1}^{o}$, where the latter refers to the interior of $P_{n+1}$. (Note that these conditions permit the set $P$ to be compact.) The set of continuous functions from $P \times R^{l}$ to $R^{m}$, to be denoted by $C\left(P \times R^{l}, R^{m}\right)$ can be endowed with the metric

$$
d(f, g)=\sum_{n=1}^{\infty} 2^{-n} \frac{\max _{P_{n} \times T_{n}}|f(p, x)-g(p, x)|}{1+\max _{P_{n} \times T_{n}}|f(p, x)-g(p, x)|},
$$


where the sequence of sets $\left\{T_{n}\right\}_{n \geq 1}$ is chosen to have the same properties in $R^{l}$ as $\left\{P_{n}\right\}_{n \geq 1}$ has in $P$. The topology generated by $d$ coincides with the topology of uniform convergence on compacta. The next two lemmas are straightforward and establish the properties we need.

Lemma 2.2. If $\bar{s}: P \times R^{l} \rightarrow R^{m}$ is continuous, so are the following functions:

(i) $\psi: R^{l} \rightarrow C\left(P \times R^{l}, R^{m}\right)$, where $\psi(t)=s_{t}$ and

(ii) $\Phi: P \times R^{l} \times C\left(P \times R^{l}, R^{m}\right) \rightarrow R^{m}$, where $\Phi(p, x, s)=s(p, x)$.

Proof: Let $M$ be any compact subset of $P \times R^{l}$ and let $t_{n}$ be a sequence in $R^{l}$ converging to $t$. It is easy to check that there is $M^{\prime}$, also compact, such that $\left(p, x+t_{n}\right)$ is in $M^{\prime}$ for all $(p, x)$ in $M$ and $t_{n}, n \geq 1$. Then $\left|s_{t_{n}}(p, x)-s_{t}(p, x)\right|=\left|\bar{s}\left(p, x+t_{n}\right)-\bar{s}(p, x+t)\right|$ can be made uniformly small on $M$ for a large enough $n$ since $\bar{s}$ is continuous and therefore uniformly continuous on $M^{\prime}$. This establishes the continuity of $\psi$.

To show that $\Phi$ is continuous, let $\left(p_{n}, x_{n}, s_{n}\right)$ converge to $(p, x, s)$. Note that

$$
\left|s_{n}\left(p_{n}, x_{n}\right)-s(p, x)\right| \leq\left|s_{n}\left(p_{n}, x_{n}\right)-s\left(p_{n}, x_{n}\right)\right|+\left|s\left(p_{n}, x_{n}\right)-s(p, x)\right| .
$$

Since the sequence $\left(p_{n}, x_{n}\right)$ is contained in a compact set, and $s_{n}$ converges to $s$ uniformly on compact sets, the first term on the right hand side can be made arbitrarily small for large enough $n$. Similarly, the continuity of $s$ guarantees that the second term on the right hand side can also be made arbitrarily small for large $n$.

Lemma 2.3. Suppose that $\bar{s}: P \times R^{l} \rightarrow R^{m}$ is uniformly continuous, and for every $p$, $\bar{s}(p, \cdot)$ is bounded. Then $\overline{\mathcal{S}}$ (the closure of $\mathcal{S}$ ) is a compact set.

Proof: Let $\left\{s_{n}\right\}_{n \geq 1}$ where $s_{n}=s_{t_{n}}$ for some $t_{n}$ in $R^{l}$ be a sequence of functions in $\mathcal{S}$. We need only show that it has a subsequence converging to a function in $C\left(P \times R^{l}, R^{m}\right)$. 
Since $s_{n}(p, x)=\bar{s}\left(p, x+t_{n}\right)$ and $\bar{s}(p, \cdot)$ is uniformly bounded, the sequence $s_{n}$ is pointwise bounded. The sequence is also equicontinuous, because $\bar{s}$ is uniformly continuous. By Ascoli's Theorem (see Royden (1968)), there is a subsequence $s_{n_{k}}$ and a function $g$ in $C\left(P \times R^{l}, R^{m}\right)$ such that $s_{n_{k}}$ tends to $g$ pointwise and uniformly on compact sets. It is now trivial to check that $d\left(s_{n_{k}}, g\right)$ tends to zero as $n_{k}$ tends to infinity.

QED

Our final result is an exact aggregation theorem, analogous to the approximate aggregation result of Proposition 2.1.

Theorem 2.4. Suppose that $\bar{s}: P \times R^{l} \rightarrow R^{m}$ is uniformly continuous, and for every $p, \bar{s}(p, \cdot)$ is bounded. Suppose also that $H: V \times R^{m} \rightarrow R^{n}$, where $V$ is a metric space, is a continuous function. Then there exists a continuous function $H^{*}: V \times P \rightarrow R^{n}$ and $a$ Borel probability measure $\mu^{*}$ on $\overline{\mathcal{S}}$ such that for all $x$ in $R^{l}$,

$$
\int_{\overline{\mathcal{S}}} H(v, \Phi(p, x, s)) d \mu^{*}=\int_{\overline{\mathcal{S}}} H(v, s(p, x)) d \mu^{*}=H^{*}(v, p) .
$$

Proof: Let $h_{n}$ be a sequence of density functions defined on $R^{l}$ such that $B\left(h_{n}\right)$ goes to zero as $n$ goes to infinity. Let $\nu_{n}$ be the probability measure on $R^{l}$ induced by $h_{n}$. This in turn induces a probability measure $\mu_{n}$ on $\overline{\mathcal{S}}$, given by $\mu_{n}\left(S^{\prime}\right)=\nu_{n}\left(\psi^{-1}\left(S^{\prime}\right)\right)$ for any Borel measurable set $S^{\prime}$ in $\overline{\mathcal{S}}$. The continuity, hence measurability, of $\psi$ (Lemma 2.2) guarantees that this definition is good. By Lemma $2.3, \overline{\mathcal{S}}$ is compact, so there is a subsequence $\mu_{n_{k}}$ which converges weakly to a measure $\mu^{*}$ (see Billingsley (1968)). We wish to show that $\int_{\overline{\mathcal{S}}} H(v, \Phi(p, x, s)) d \mu^{*}=\int_{\overline{\mathcal{S}}} H(v, \Phi(p, \tilde{x}, s)) d \mu^{*}$ for all $x$ and $\tilde{x}$ in $R^{l}$. 
To see this we note that $\left|\int_{\overline{\mathcal{S}}} H(v, \Phi(p, x, s))-H(v, \Phi(p, \tilde{x}, s)) d \mu^{*}\right|$ is less than

$$
\begin{gathered}
\left|\int_{\overline{\mathcal{S}}} H(v, \Phi(p, x, s)) d \mu^{*}-\int_{\overline{\mathcal{S}}} H(v, \Phi(p, x, s)) d \mu_{n}\right|+\left|\int_{\overline{\mathcal{S}}} H(v, \Phi(p, x, s)) d \mu_{n}-\int_{\overline{\mathcal{S}}} H(v, \Phi(p, \tilde{x}, s)) d \mu_{n}\right| \\
+\left|\int_{\overline{\mathcal{S}}} H(v, \Phi(p, \tilde{x}, s)) d \mu_{n}-\int_{\overline{\mathcal{S}}} H(v, \Phi(p, \tilde{x}, s)) d \mu^{*}\right| .
\end{gathered}
$$

The function $H(v, \Phi(p, x, \cdot))$ is uniformly continuous and bounded when restricted to the compact set $\overline{\mathcal{S}}$ (by Lemmas 2.2 and 2.3 ), so the weak convergence of $\mu_{n_{k}}$ to $\mu^{*}$ guarantees that when $n_{k}$ is arbitrarily large, the first term (and similarly the last term) will be arbitrarily small. Note that the second term is just

$$
\left|\int H(v, \bar{s}(p, x+t)) h_{n_{k}}(t) d t-\int H(v, \bar{s}(p, \tilde{x}+t)) h_{n_{k}}(t) d t\right|
$$

Proposition 2.1 guarantees that this term will be arbitrarily small when $n_{k}$ becomes large since $H(v, \bar{s}(p, \cdot))$ is a measurable and bounded function . Thus we have established our claim: the value of $\int_{\overline{\mathcal{S}}} H(v, \Phi(p, x, s)) d \mu^{*}$ is independent of $x$, and we may write it as $H^{*}(v, p)$.

We need only show that $H^{*}$ is continuous. Let $\left(v_{n}, p_{n}\right)$ tend to $(v, p)$. The sequence of functions on $\overline{\mathcal{S}},\left\{H\left(v_{n}, \Phi\left(p_{n}, x, \cdot\right)\right\}_{n \geq 1}\right.$ is uniformly bounded since $H$ and $\Phi$ are continuous and $\overline{\mathcal{S}}$ is a compact set. By the dominated convergence theorem, $\int_{\overline{\mathcal{S}}} H\left(v_{n}, \Phi\left(p_{n}, x, s\right)\right) d \mu^{*}$ converges to $\int_{\overline{\mathcal{S}}} H\left(v, \Phi(p, x, s) d \mu^{*}\right.$, but this is equivalent to saying that $H^{*}\left(v_{n}, p_{n}\right)$ converges to $H^{*}(v, p)$.

If we choose $V$ to be a singleton and $H$ to satisfy $H(v, y)=y$ for all $y$ in $R^{m}$, we obtain the following corollary.

Corollary 2.5. Suppose that $\bar{s}: P \times R^{l} \rightarrow R^{m}$ is uniformly continuous, and for every $p, \bar{s}(p, \cdot)$ is bounded. Then there exists a continuous function $s^{*}: P \rightarrow R^{m}$ and a Borel 
probability measure $\mu^{*}$ on $\overline{\mathcal{S}}$ such that for all $x$ in $R^{l}, \int_{\overline{\mathcal{S}}} s(p, x) d \mu^{*}=s^{*}(p)$.

\section{Some Examples}

Grandmont (1992) develops a model of an exchange economy where the excess demand function obeys gross substitubility. In essence, he considers an economy consisting of groups of agents where the distribution of demand behavior in each group is such that the group's mean demand has an approximate Cobb-Douglas behavior; it is then easy to check that an economy consisting of such groups will have an excess demand function which obeys gross substitubility (from which one obtains the uniqueness and stability of equilibrium and other nice properties). Our first example applies the results of the last section to show how Cobb-Douglas behavior, in both its approximate and exact form, can be obtained in aggregate.

Example 1. Let $\bar{f}: R_{++}^{l} \rightarrow R_{++}^{l}$ be a function satisfying $q \cdot \bar{f}(q)=\bar{w}$, where $\bar{w}$ is some positive scalar. We interpret $\bar{f}(q)$ as the demand of an agent with income $\bar{w}$ and facing price $q$. The expenditure share function of $\bar{f}$ is $\bar{s}: R^{l} \rightarrow R_{++}^{l}$, defined by $\bar{s}^{i}(x)=e^{x^{i}} \bar{f}^{i}\left(e^{x^{1}}, e^{x^{2}}, \ldots, e^{x^{l}}\right) / \bar{w}$ for $i=1,2, \ldots, l$. So $\bar{s}^{i}(x)$ is the share of expenditure devoted to good $i$ when the price is $\left(e^{x^{1}}, e^{x^{2}}, \ldots, e^{x^{l}}\right)$. Note that $\sum_{i=1}^{l} \bar{s}^{i}(x) \equiv 1$. With $\bar{f}$ and given $t$ in $R^{l}$, we may define $f_{t}$ by $f_{t}^{i}(q)=e^{t^{i}} \bar{f}^{i}\left(q^{1} e^{t^{1}}, q^{2} e^{t^{2}}, \ldots, q^{l} e^{t^{l}}\right)$ for $i=1,2, \ldots, l$. Following the literature, we will refer to $f_{t}$ as an affine transformation of $\bar{f}$. It is trivial to check that the expenditure share function of $\bar{f}_{t}$, which we denote by $s_{t}$, is related to $\bar{s}$ by $s_{t}(x)=\bar{s}(t+x)$.

The special value of affine transformations lies in the fact that it preserves standard 
rationality properties that $\bar{f}$ might satisfy. In particular the following claims are easy to check: (a) if $\bar{f}$ is generated by a utility function, so is $f_{t}$; (b) if $\bar{f}$ satisfies the weak axiom, so will $f_{t}$; and (c) if $\bar{f}$ satisfies the law of demand, so will $f_{t}{ }^{3}$

Consider a market consisting of agents with the same income $\bar{w}$ and expenditure share functions drawn from the set $\mathcal{S}$ (as defined in Section 2). If the distribution of $t$ is governed by the density $h: R^{l} \rightarrow R_{+}$, then this market's mean demand at price $q=\left(e^{x^{1}}, e^{x^{2}}, \ldots, e^{x^{l}}\right)$ is $\int f_{t}(q) h(t) d t$, while the mean expenditure share on good $i$ is $s_{h}^{i}(x)=\int s_{t}^{i}(x) h(t) d t$. Proposition 2.1 and the discussion following it tells us that there exists density functions $h$ such that $s_{h}(x)$ is approximately independent of $x$. In other words, the distribution of demand behavior could be such that the mean expenditure devoted to any good is approximately independent of prices. This is the central claim in Grandmont (1992).

To obtain an exact version of this result, we assume that agents' expenditure share functions are drawn from a slightly larger set, $\overline{\mathcal{S}}$. Agents in this market all have income $\bar{w}$, so to each $s$ in $\overline{\mathcal{S}}$ is associated a demand function $f_{s}: R_{++}^{l} \rightarrow R_{+}^{l}$ where $f_{s}^{i}(q)=$ $\bar{w} s^{i}\left(\ln q^{1}, \ln q^{2}, \ldots, \ln q^{l}\right) / q^{i}$. Clearly, if $s=s_{t}$ for some $t$, then $f_{s}=f_{t}$. (It is clear that notation has been abused.) When $\bar{f}$ satisfies a rationality property like (a), (b) or (c), $f_{s}$ need not satisfy the property exactly, but on any compact set of strictly positive prices, it will be arbitrarily close to a function $f_{s^{\prime}}$ which does satisfy the property. This follows easily from the fact that the members of $\overline{\mathcal{S}}$ are either translations of $\bar{s}$ or arbitrarily close, on compact sets, to translations of $\bar{s}$. If the distribution of characteristics in this market is governed by a Borel probability measure $\mu$ on $\overline{\mathcal{S}}$, then market demand at price $q$ is $F(q)=\int_{\overline{\mathcal{S}}} f_{s}(q) d \mu$. 
The function $\bar{s}$ is bounded by definition. If in addition it is uniformly continuous, then Corollary 2.5 tell us that there is a probability measure $\mu^{*}$ such that $\int_{\overline{\mathcal{S}}} s(x) d \mu^{*}=$ $s^{*}$, where $\sum_{i=1}^{l} s^{* i}=1$. In other words, the mean expenditure devoted to any good is independent of the prevailing price. More formally, $F^{i}(q)=s^{* i} \bar{w} / q^{i}$, which coincides with the demand at price $q$ and income $\bar{w}$ of an agent with the Cobb-Douglas utility function $u(x)=\sum_{i=1}^{l} s^{* i} \ln x^{i}$.

The first model of exact Cobb-Douglas aggregation is in Giraud and Maret (2001). The assumptions we make here are generally weaker than theirs, but the stronger assumptions in that paper allow for some stronger conclusions. Notice that our result allows the measure $\mu^{*}$ to be concentrated on just a few elements of $\overline{\mathcal{S}}$ though, as we shall see in the next section, there is a straightforward way of guaranteeing that it is not concentrated on just a single function in that set. Giraud and Maret (2001) identify conditions which guarantee that $\mu^{*}$ is spread out across many elements. Loosely speaking, they show that if $\mathcal{S}$ is compact in the sup norm, then $\mu^{*}$ can be chosen to assign a non-zero measure to open subsets of $\mathcal{S}$. Note that the assumption made in this case imposes a strong restriction on the generating function $\bar{s}$. Giraud and Maret (2001) also show that $\mu^{*}$ can be approximated by measures of finite support in a way which leads to approximate Cobb-Douglas behavior for the market. (Related results on finite approximations can also be found in Quah (1997).)

Example 2. It is not always essential that mean demand obeys Cobb-Douglas behavior. We may be interested in a weaker property. Quah (1997) considers a market where the distribution of characteristics are such that its mean demand is approximately linear in income; in other words, market demand takes on homothetic-like (but not necessarily Cobb- 
Douglas-like) properties. ${ }^{4}$ Once again, in the right mathematical context, this could be sharpened to exact linearity in income.

Let $\bar{g}: P \times R_{+} \rightarrow R_{+}^{l}$ be a function satisfying $p \cdot \bar{g}(p, w)=w$. We interpret $\tilde{g}$ as a demand function, in which case, $P$ (a subset of $R_{++}^{l}$ ) is the set of prices under consideration. The expenditure share function $\bar{s}: P \times R \rightarrow R_{++}^{l}$ gives the share of expenditure devoted to each good, so $\bar{s}^{i}(p, x)=p^{i} e^{-x} \bar{g}^{i}\left(p, e^{x}\right)$ for $i=1,2, \ldots, l$. Given $\bar{g}$, we may define $g_{t}: P \times R_{+} \rightarrow R_{+}^{l}$ by $g_{t}(p, w)=e^{-t} \bar{g}\left(p, w e^{t}\right)$. It is usual to refer to $g_{t}$ a homothetic transformation of $\bar{g}$; if $\bar{g}$ is homogeneous of degree zero, then a homothetic transformation is a special case of an affine transformation. The expenditure share function of $g_{t}$, which we denote by $s_{t}$, is related to $\bar{s}$ by $s_{t}(p, x)=\bar{s}(p, x+t)$. Once again, it is easy to check that if $\bar{g}$ satisfies the rationality properties (a), (b) or (c), so will $g_{t}$.

Consider a market consisting of agents with the same income, and with expenditure share functions drawn from the set $\mathcal{S}$. If the distribution of $t$ is governed by the density $h: R \rightarrow R_{+}$, then this market's mean expenditure share at price $p$ and when each agent has an income of $e^{x}$ is $s_{h}(p, x)$. Proposition 2.1 and the discussion following it tells us that there exists density functions $h$ such that $s_{h}(p, x)$ is approximately independent of $x$. In other words, the mean expenditure devoted to any good is approximately independent of income or mean demand becomes approximately homothetic.

For an exact version of this result, we assume as before that all agents have the same income, while expenditure share functions are drawn from $\overline{\mathcal{S}}$. To each $s$ in $\overline{\mathcal{S}}$ we may associate a demand function $g_{s}$ in the obvious way. A market where all agents face the price $p$ and have the same income $w$, and where expenditure share functions are drawn from 
the set $\overline{\mathcal{S}}$ according to the probability measure $\mu$ will have aggregate demand of $G(p, w)=$ $\int_{\overline{\mathcal{S}}} g_{s}(p, w) d \mu$. The average expenditure share on good $i$ when $w=e^{x}$ is $\int_{\overline{\mathcal{S}}} s^{i}(p, x) d \mu$. The function $\bar{s}$ is bounded by definition. If in addition it is uniformly continuous, then Corollary 2.5 tells us that there is a probability measure $\mu^{*}$ such that $\int_{\overline{\mathcal{S}}} s(p, x) d \mu^{*}=s^{*}(p)$, where $\sum_{i=1}^{l} s^{* i}(p)=1$. In other words, the share of expenditure devoted to each good is independent of the income $e^{x}$. More formally, $G^{i}(p, w)=s^{* i}(p) w / p^{i}$ for $1 \leq i \leq l$.

In the next example, we consider a market where agents individually obey the weak axiom and where a suitable distribution of characteristics will guarantee that market demand satisfies a stronger property, namely, the law of demand.

Example 3. We retain the setup of Example 2. We impose the additional assumption that $P$ is open and convex set in $R_{++}^{l}$ and that $\bar{g}$ is $C^{1}$, so its associated expenditure share function $\bar{s}$ is also $C^{1}$. Consider the map $\bar{\sigma}$ from $P \times R$ to $R^{2 l+l^{2}}$ given by

$$
(p, x) \rightarrow\left(\bar{s}(p, x), \frac{\partial \bar{s}^{i}}{\partial p^{j}}(p, x)_{1 \leq i, j \leq l}, \frac{\partial \bar{s}^{i}}{\partial x}(p, x)_{1 \leq i \leq l}\right)
$$

Define $\sigma_{t}$ by $\sigma_{t}(p, x)=\bar{\sigma}(p, x+t)$ and the set $\Sigma=\left\{\sigma_{t}: t \in R\right\}$. We denote its closure by $\bar{\Sigma}$. Provided $\bar{\sigma}$ is uniformly continuous and $\bar{\sigma}(p, \cdot)$ is bounded for all $p$ in $P$, the set $\bar{\Sigma}$ is compact (Lemma 2.3). Furthermore, for every $\sigma$ in $\bar{\Sigma}$ there is a $C^{1}$ function $s: P \times R \rightarrow R_{+}^{l}$ such that

$$
\sigma(p, x)=\left(s(p, x), \frac{\partial s^{i}}{\partial p^{j}}(p, x)_{1 \leq i, j \leq l}, \frac{\partial s^{i}}{\partial x}(p, x)_{1 \leq i \leq l}\right) .
$$

To every $\sigma$ in $\bar{\Sigma}$ we can associate the demand function $g_{\sigma}: P \times R_{+} \rightarrow R_{++}^{l}$ in the usual way, namely, $g_{\sigma}^{i}(p, w)=\sigma^{i}(p, \ln w) w / p^{i}$ for $1 \leq i \leq l$. The function $g_{\sigma}$ is $C^{1}$ and its derivative matrix with respect to price, which we will denote by $\partial_{p} g_{\sigma}(p, w)$, can be 
decomposed into the substitution and income effect matrices. If $\bar{g}$ satisfies the weak axiom, its substitution effect matrix is negative semi-definite; it is not hard to check that this implies that the substitution effect matrix of $g_{\sigma}$ is also negative semi-definite.

Consider a market where $\sigma$ is drawn from $\bar{\Sigma}$ according to the Borel probability measure $\mu$. Market demand is $G(p, w)=\int_{\bar{\Sigma}} g_{\sigma}(p, w) d \mu$. We wish to show that there is $\mu^{*}$ such that, when $\mu=\mu^{*}$, the matrix $\partial_{p} G(p, w)$ is negative semi-definite for all $(p, w)$ in $P \times R_{+}$. This will guarantee that $G$ satisfies the weak law of demand, i.e., $\left(p-p^{\prime}\right) \cdot\left(G(p, w)-G\left(p^{\prime}, w\right)\right) \leq 0$ for all $(p, w)$ and $\left(p^{\prime}, w\right)$ in $P \times R_{+}$.

Each $\partial_{p} g_{\sigma}(p, w)$ can be decomposed into its substitution and income effect matrices, with the former being negative semi-definite, so all that we need to do is to show that the aggregate of income effect matrices

$$
I(p, w)=\int_{\bar{\Sigma}} g_{\sigma}(p, w)\left(\frac{\partial g_{\sigma}}{\partial w}(p, w)\right)^{T} d \mu
$$

is a positive semi-definite matrix.

To see this, we let $V=R^{l}$ and consider the map $H: R^{l} \times R^{2 l+l^{2}} \rightarrow R$ by $H(v, \theta)=(v \cdot \hat{\theta})^{2}$ where $\hat{\theta}$ refers to the first $l$ entries in $\theta . H$ is clearly a continuous function, so Theorem 2.4 tells us that there is $H^{*}: R^{l} \times P \rightarrow R$ such that

$$
\int_{\bar{\Sigma}} H(v, \Phi(p, x, \sigma)) d \mu^{*}=\int_{\bar{\Sigma}}[v \cdot s(p, x)]^{2} d \mu^{*}=H^{*}(v, p) \geq 0
$$

where $s(p, x)$ refers to the first $l$ entries of $\sigma(p, x)$. Therefore, with $\mu=\mu^{*}$, and writing $v=\left(p^{1} u^{1}, \ldots, p^{l} u^{l}\right)$,

$$
v \cdot I(p, w) v=\frac{1}{2} \frac{\partial}{\partial w} \int_{\bar{\Sigma}}\left[v \cdot g_{\sigma}(p, w)\right]^{2} d \mu^{*}
$$




$$
\begin{aligned}
& =\frac{1}{2} \frac{\partial}{\partial w}\left(w^{2} \int_{\bar{\Sigma}}\left[\frac{v \cdot g_{\sigma}(p, w)}{w}\right]^{2} d \mu^{*}\right) \\
& =H^{*}(u, p) w \geq 0 .
\end{aligned}
$$

So we have shown that there is a distribution over $\bar{\Sigma}$ such that market demand $G$ satisfies the law of demand. In fact, it satisfies the stronger property that the matrix $I(p, w)$ is negative semi-definite. This property is known as increasing dispersion and empirical tests of the law of demand typically test this stronger property. (See W. Hildenbrand (1994) for some empirical work and Jerison (1999) for a theoretical discussion of this property and its variants.) It is not hard to check that by suitably re-defining $H$ we can obtain a measure $\mu^{*}$ such that $G$ is both monotonic and a linear function of income (as in Example 2). In Quah's (1997) model of an exchange or production economy satisfying the law of demand, the economy consists of groups of households in which both these properties, in their approximate versions, hold simultaneously for each group.

\section{Guaranteeing Heterosis}

The models presented in the last section have usually been understood as examples of the heterotic phenomenon at work. So in Example 2, agents need not individually have demand functions which are linear in income; it is the distribution of demand behavior in the market which leads to that property. This is a valid interpretation, provided $\bar{s}$ satisfies certain conditions.

It is best to begin with cases where such an interpretation is problematic. We confine our observations to the model described in Example 2, though these observations apply just as aptly to Example 1. In Example 2, one trivial situation which is clearly not an 
example of heterosis, indeed not even an example of heterogeneity, is when $\tilde{g}$ is itself linear in income; then $\bar{s}$ will be independent of $x$ and $\mathcal{S}$ is a singleton consisting of $\bar{s}$. In this case, Proposition 2.1, Theorem 2.4, and Corollary 2.5 are all trivially true, but the market, which consists of essentially just one very well-behaved agent, is clearly not heterogenous in any reasonable sense of the word.

A less obvious and more interesting situation has been pointed out by B. de Villemeur (1998) and K. Hildenbrand (1998). The essence of what they are saying could be seen in Figure 1, which shows the graph of $\bar{s}^{i}(p, \cdot)$. Suppose we are interested in the behavior of $s_{h}^{i}(p, x)$ for $x$ in some compact interval $X$. From Proposition 2.1 and the subsequent discussion we know that the value of $s_{h}^{i}(p, x)$ in $X$ becomes increasingly independent of $x$ as the density of $h$ becomes increasingly flat. However, in this case, there is a sense in which it is misleading to say that this approximate independence on $x$ is caused by heterogeneity. The reason is that as $h$ becomes increasingly flat, it places most of its weight on the values of $t$ that are either very large or very small. If the function $\bar{s}$ has limits as $x$ goes to positive or negative infinity, the behavior of $s_{t}$, at least within the interval $X$, becomes itself increasingly independent of $x$ for very large or small $t$, since $s_{t}(x)=\bar{s}(x+t)$ (see Figure 1). In other words, market behavior in the set $X$, as represented by $s_{h}(p, x)$, becomes increasingly independent of $x$, but only because the agents who make up that market increasingly take on the same property in $X$. Such a market can be described as heterogenous in the sense that it is composed of agents with different expenditure share functions, but, provided we judge agents' demand only by its behavior in $X$, this is clearly not an example of heterosis at work. 
There is another way of seeing essentially the same thing. In Figure $1, \bar{s}^{i}(p, \cdot)$ has a limit of $L^{i}$ as $x$ goes to infinity and a limit of $M^{i}$ as $x$ goes to minus infinity. It is trivial to check that the constant functions $\hat{s}^{i}(p, x) \equiv L^{i}$ and $\tilde{s}^{i}(p, x) \equiv M^{i}$ are in $\overline{\mathcal{S}}$. So there certainly is a measure $\mu^{*}$ such that $\int_{\overline{\mathcal{S}}} s^{i}(p, x) d \mu^{*}$ is independent of $x$ : simply choose a measure which places all its weight on $\hat{s}^{i}(p, \cdot)$ and $\tilde{s}^{i}(p, \cdot)$. Once again there is heterogeneity but no heterosis.

On the other hand, the function $\bar{s}^{i}(p, \cdot)$ can look like the one depicted in Figure 2. In this case, it is clear that $s_{t}^{i}(p, x)$ will not be approximately independent of $x$ in $X$ for any $t$. Then if $s_{h}^{i}(p, x)$ is approximately independent of $x$ - as it will be if $h$ is sufficiently flat - this phenomenon must arise from the distribution of $t$. In other words, as $x$ is increased, $s_{t}^{i}(p, x)$ rises for some values of $t$ and falls for other values in a way which approximately cancels out. We may say that the agents exhibit a complementary or sign-balancing heterogeneity in their demand behavior. (The terms are loosely borrowed from B. de Villemeur (2001) and W. Hildenbrand and A. Kneip (1999).) This is a bona fide model of heterosis.

If we wish to guarantee that we have a model of complementary heterogeneity, then we should impose conditions on $\bar{s}$ so that it looks like Figure 2 and not like Figure 1. This is straightforward. We say that a function $H: R^{l} \rightarrow R^{m}$ satisfies uniform variation if there is $\delta$ and $\epsilon$ such that at any point $x$ in $R^{l}$, the compact neighborhood around $x$ with radius $\delta$ contains elements $x^{\prime}$ and $x^{\prime \prime}$ satisfying $\left|H\left(x^{\prime \prime}\right)-H\left(x^{\prime}\right)\right|>\epsilon$. The property simply guarantees that within sets of sufficient size, there will be a uniformly large variation in the function $H$. A family $\mathcal{H}$ of functions from $R^{l}$ to $R^{m}$ satisfies equi-variation if there is $\delta$ and $\epsilon$ such that at any point $x$ in $R^{l}$ and for any $H$ in $\mathcal{H}$, the compact neighborhood around $x$ with 
radius $\delta$ contains elements $x^{\prime}$ and $x^{\prime \prime}$ (which depends on $H$ ) satisfying $\left|H\left(x^{\prime \prime}\right)-H\left(x^{\prime}\right)\right|>\epsilon$. The next lemma has a completely trivial proof which we will omit.

Lemma 4.1 If $\bar{s}(p, \cdot)$ satisfies uniform variation, then the set $\{s(p, \cdot): s \in \overline{\mathcal{S}}\}$ satisfies equi-variation.

The import of this observation is that provided $\bar{s}(p, \cdot)$ satisfies uniform variation for some $p$ in $P$, the functions $s(p, \cdot)$, where $s$ is in $\overline{\mathcal{S}}$, are never independent of $x$. So that when we find some $\mu^{*}$ such that, for all $p$ in $P, \int_{\overline{\mathcal{S}}} s(p, x) d \mu^{*}$ is independent of $x$, this is indeed a manifestation of heterosis arising from complementary heterogeneity.

A difficulty with this uniform variation requirement has been raised by some researchers (for example, K. Hildenband (1998)): they observe that it is implausible (though not irrational in the sense of violating the standard rationality properties) for expenditure shares to behave that way, where there is no nice limiting behavior as income becomes very small or very large. In other words, any interpretation of the models of market demand given in Section 3 as models of complementary heterogeneity requires implausible assumptions. We do not share this view. When one is modelling demand behavior, it is not usually demand behavior in the entire price-income space that is being modelled, but rather the behavior of demand in some pre-determined set of prices and income that is bounded and bounded away from the margins (like the set $X$ in Figure 1). One may model demand in this set by the elements of $\mathcal{S}$ or its closure; whether or not the model is realistic depends on whether the elements of $\mathcal{S}$ (or $\overline{\mathcal{S}}$ ) adequately represent the variety of demand behavior in that set. It is on this basis that one should judge the suitability of the generating function $\bar{s}$; the realism or otherwise of $\bar{s}$ as a description of a consumer's behavior over the entire price-income 
space is not relevant in itself. ${ }^{5,6}$

CNRS UMR 7522, Bureau d'Economie Theorique et Appliquee, 61, avenue de la Foret Noire,67000,Strasbourg, France.; ggiraud@cournot.u-strasbg.fr

and

St Hugh's College, Oxford, OX2 6LE.U.K.; john.quah@economics.ox.ac.uk

\section{REFERENCES}

Billette De Villemeur, E. (2001): "Behavioral Complementarity (not Heterogeneity) Causes the Law of Demand," Personal Manuscript.

Billingsley, P. (1968): Convergence of Probability Measures. New York: Wiley.

Giraud, G. And I. Maret (2001): "Behavioral Heterogeneity in Large Economies," mimeo.

Grandmont, J.-M. (1992): "Transformations of the commodity space, behavioral heterogeneity, and the aggregation problem," J. Econ Theory, 57, 1-35.

Hildenbrand, K. (1998): “On J. M. Grandmont's Modelling of Behavioral Heterogeneity," Bonn discussion Paper, A-580.

Hildenbrand, W. (1974): Core and Equilibria of a Large Economy. Princeton: Princeton University Press.

— (1983): "On the Law of Demand," Econometrica, Vol. 51, 997-1019.

— (1994): Market Demand. Princeton: Princeton University Press. 
Hildenbrand, W. And A. KneIP (1999): “On Behavioral Heteorgeneity,” Bonn Discussion Paper, A-589.

JERISON, M. (1999): "Dispersed excess demands, the weak axiom and uniqueness of equilibrium," J. Math Econ., Vol. 31, 15-48.

QuAH, J. K.-H. (1997): "The Law of Demand when Income is Price Dependent," Econometrica, Vol. 65, No. 6, 1421-1442.

QuAH, J. K.-H. (2001): "Demand is Heterogenous in Grandmont's Model," Nuffield College Working Paper, W12.

Royden, H. L. (1968): Real Analysis, 2nd. edition. London: Macmillan. 


\section{Footnotes}

1. 'Heterosis' is not a new word, though it is fairly obscure. The Oxford English Dictionary gives it a few different meanings, but its most common use (and apparently even this use is not that common) is in biology, where it means hybrid vigor. The meaning we are giving to this word is different, but it follows just as naturally from its Greek roots. Of course, economists have been known to borrow words from other disciplines: 'hysteresis' being just one example.

2. The crucial assumption in Hildenbrand and Kneip's (1999) interpretation of Grandmont's result is that the expenditure share function generating the affine class has, in a certain sense, finitely many turning points (Assumption 3 in their paper). For a more detailed discussion of these issues see Quah (2001).

3. A function $f: R_{++}^{l} \times R_{+} \rightarrow R_{++}^{l}$ satisfies the weak axiom if $p \cdot f\left(p^{\prime}, w^{\prime}\right) \leq p \cdot f(p, w)$ implies $p^{\prime} \cdot f(p, w)>p^{\prime} \cdot f\left(p^{\prime}, w^{\prime}\right)$. It satisfies monotonicity or the law of demand if $\left(p-p^{\prime}\right) \cdot\left(f(p, w)-f\left(p^{\prime}, w\right)\right)<0$. For a discussion of these concepts see Mas-Colell et al (1996). In the case of (a), if $\bar{f}$ is generated by $u$, then $f_{t}$ is generated by $u_{t}$, where $u_{t}(x)=u\left(e^{-t^{1}} x^{1}, e^{-t^{2}} x^{2}, \ldots, e^{-t^{l}} x^{l}\right)$.

4. A preference generates a demand function which is linear in income if and only if it is homothetic. For this reason and for convenience, we use the terms 'homotheticity' and 'linearity in income' interchangeably, but the reader should bear in mind that even when market demand is linear in income it need not be generated by a preference.

5. Part of this discussion was first reported in Quah (2001).

6. Since a homothetic or affine transformation of a demand function will inherit any im- 
plausible boundary behavior it has, excluding the situation depicted in Figure 2 may be reasonable if a model assumes agents to have demand functions which coincide with these transformations on the entire set of prices and incomes. Indeed, this assumption is made in Grandmont (1992) and Quah (1997), but while it simplifies the exposition, in neither case is the assumption essential. The authors were principally interested in the global uniqueness and stability of the equilibrium price. For simplicity, consider an exchange economy. Then the strategy is as follows. Firstly, some assumptions are made (and there are many variations, beyond the ones supplied by those authors) which guarantee that the equilibrium prices lie within some compact set of strictly positive prices, $\mathrm{P}$; given $P$, and given the endowments of the agents, we can find a compact interval of strictly positive incomes which agents in the model can achieve given the prices in $P$. Secondly, in the set $P \times W$, but not necessarily outside that set, agents have demand functions which belong to homothetic classes in the case of Quah (1997) and affine classes in the case of Grandmont (1992). Suitable distributional assumptions within these classes plus other assumptions then guarantee an aggregate demand structure nice enough to ensure uniqueness and stability of the equilibrium price. 


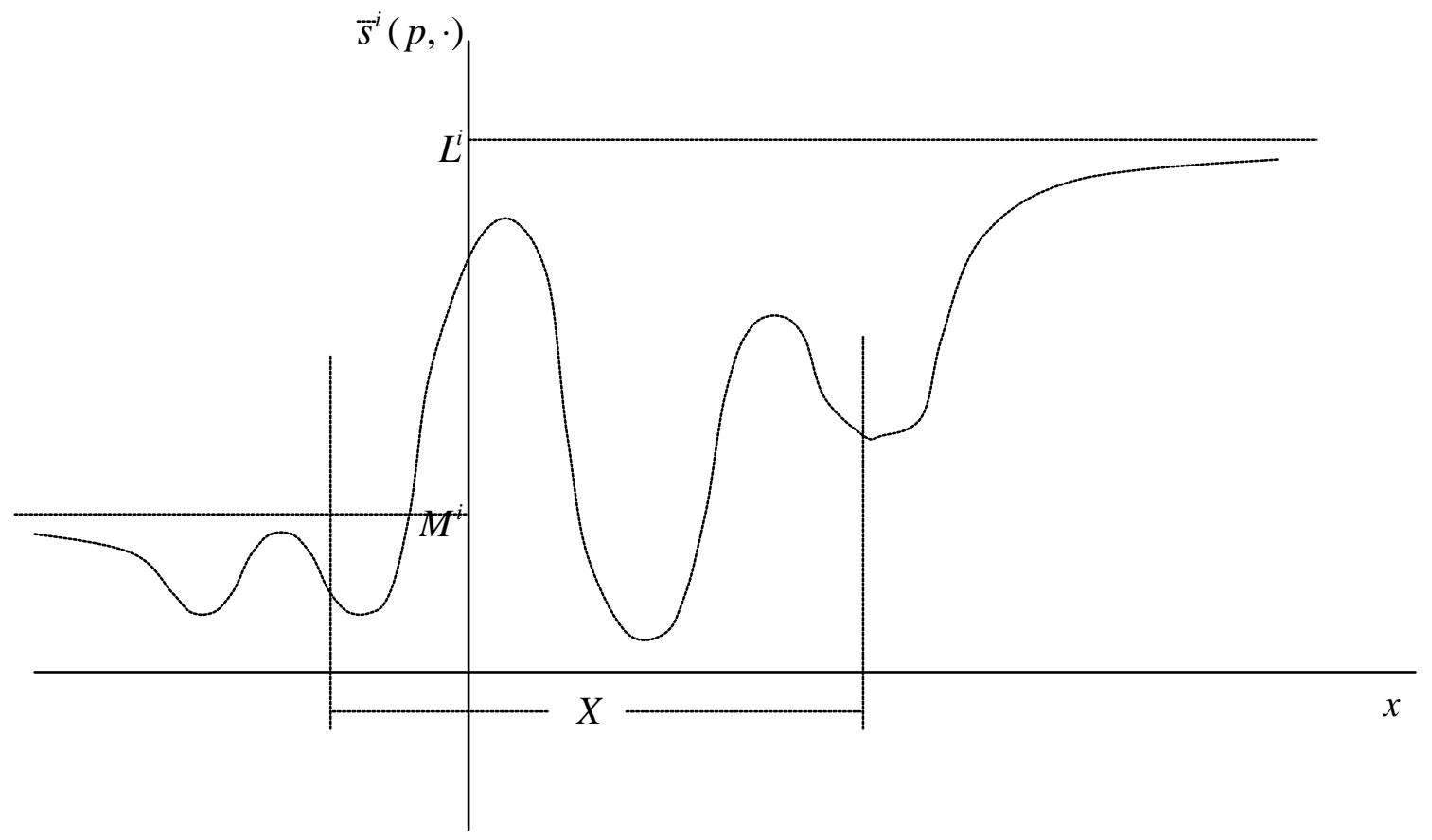

FIGURE 1

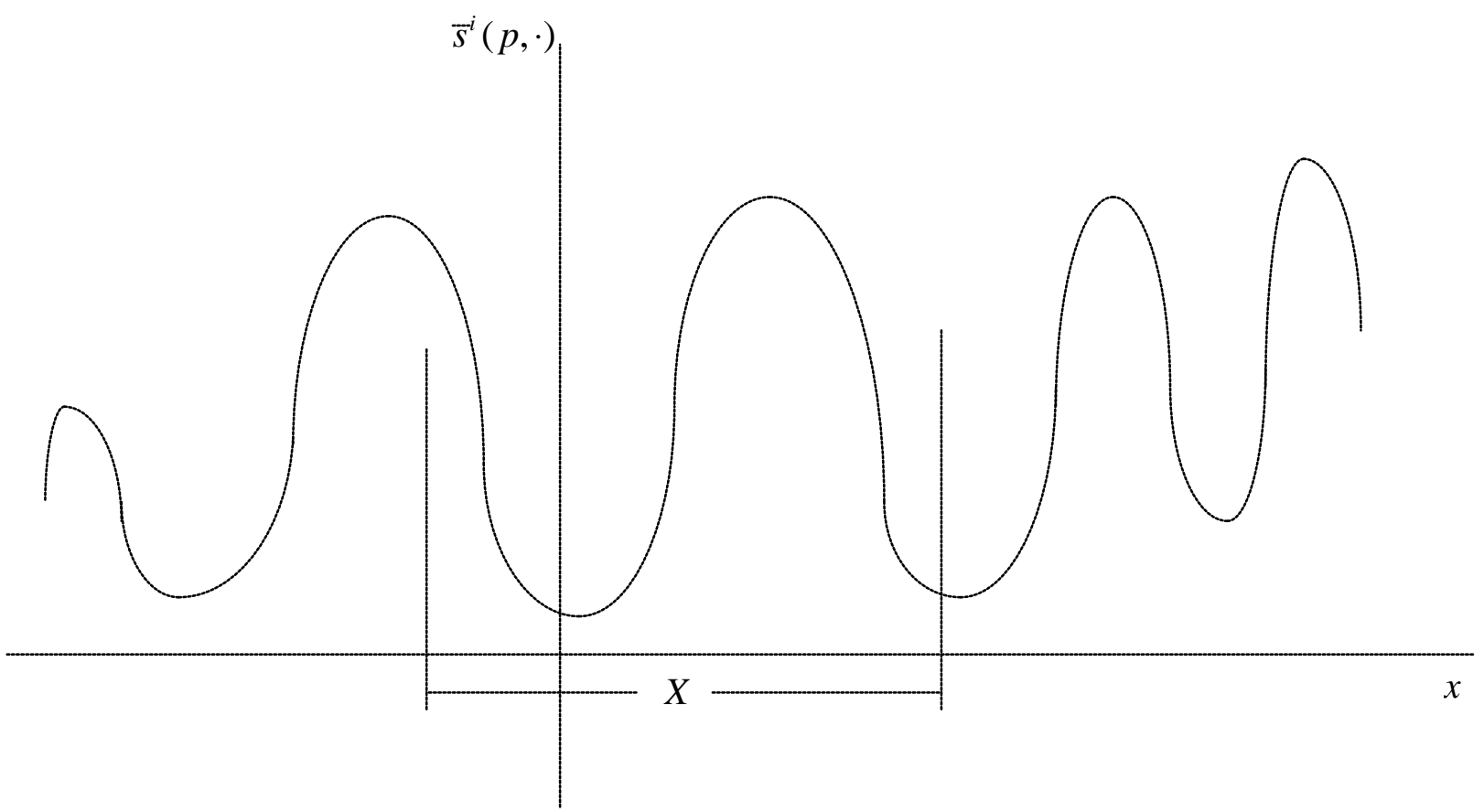

FIGURE 2 